\title{
HIGH POWER SOLITON GENERATION USING HYBRID NONLINEAR TRANSMISSION LINES*
}

\author{
L. P. Silva Neto ${ }^{\xi}$, J. O. Rossi ${ }^{1}$, J. J. Barroso ${ }^{1}$, E. Schamiloglu ${ }^{2}$ \\ Federal University of São Paulo, PO Box 1234, Mail Stop H-567 \\ São José dos Campos, São Paulo, Brazil
}

\section{Abstract}

Nonlinear Transmission lines (NLTLs) have been studied for high power RF generation with good prospects of applications in telecommand systems of small satellites (Cubesats), pulse radars for remote sensing (SARs) and disruption of communications in battlefield, [1], [2] for instance. Usually NLTLs employ barium titanate (BT) ceramic-based capacitors or ferrite bead inductor as nonlinear elements, denominated as capacitive or inductive lines, respectively. On the other hand, a configuration that employs both nonlinear elements (LC), known as hybrid line, is an efficient approach for exciting soliton oscillations more easily than inductive or capacitive lines. Other interesting aspect is that numerous publications on NLTL with only one linear component are easily found in the literature whereas just a few on hybrid lines and only one considering line experimental tests. In view of that, in this work a 30section hybrid NLTL built using inductors and capacitors of strong nonlinearity $(2.2 \mathrm{nF}$ BT ceramic capacitors and $10 \mu \mathrm{H}$ ferrite bead inductors) will be described. For the test, the line is fed by a negative input pump pulse generated by a $1 \mathrm{kV}$ discharge of a $0.75 \mu \mathrm{F}$ storage capacitor via a fast $50 \mathrm{~ns}$ switching system composed by an IGBT switch and its gate circuit driver. In the hybrid line tests, the soliton generation packet obtained on the middle section had a frequency of the order $33.0 \mathrm{MHz}$, peak power of $13.0 \mathrm{~kW}$ and voltage modulation depth (VMD) of around $700 \mathrm{~V}$. For each single shot, approximately $10 \mathrm{RF}$ cycles with small damping were noted. The main conclusion from this experiment is that with an improved proper design hybrid lumped NLTLs may be used to achieve RF in the range of 100-200 MHz.

\section{I.INTRODUCTION}

Nonlinear transmission lines have been studied to be applied in different types of systems such as battlefield communications disruption, satellite communications, radars and biomedical applications [1], [2]. Nowadays we find many works using a capacitor or inductor as nonlinear element in the line, but few are found on hybrid line, as for example only one about line experimental tests [3].

In Fig. 1 it is shown the basic schematic of a hybrid nonlinear transmission line (HNLTL) using capacitors and inductors with nonlinear behavior as a function of the voltage and current respectively.

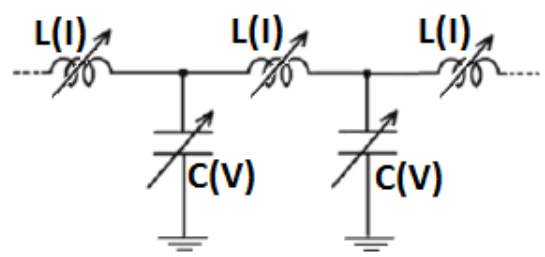

Figure 1. A hybrid nonlinear transmission line (HNLTL).

In this work, a hybrid nonlinear transmission line (HNLTL) with 30 sections was built using $2.2 \mathrm{nF}$ commercial ceramic capacitors and $10 \mu \mathrm{H}$ ferrite beads, both as nonlinear elements.

\section{METHODOLOGY}

As discussed in the introduction, we use both capacitor and inductor with nonlinear behavior to build a HNLTL. In this work, a commercial-off-the-shelf (COTS) ceramic capacitor with strong nonlinearity from Murata manufacturer, part number DEBF33A222ZA2B, was selected for line construction. Based on US EIA standards this capacitor selected is $2.2 \mathrm{nF}$ with $\mathrm{Y} 5 \mathrm{~V}$ dielectric (capacitance tolerance of $+22 \%$ to $-82 \%$ ) over a temperature range between $-30^{\circ} \mathrm{C}$ and $+85^{\circ} \mathrm{C}$. To measure the capacitance as a function of the applied voltage, the capacitor is polarized via a HV DC power supply and its capacitance measured as a function of the applied voltage using a C-meter isolated by linear capacitor of high capacitance [4]. The $\mathrm{C}-\mathrm{V}$ curve shown in Fig. 2 gives a capacitance variation of the order of $85 \%$ near the breakdown voltage.

\footnotetext{
* Work supported in part by SOARD-USAF under contract number FA9550-14-1-0133

$\xi$ email: silvaneto007@yahoo.com.br

${ }^{I}$ National Institute for Space Research, Associated Plasma Laboratory, São José dos Campos, Brazil

${ }^{2}$ University of New Mexico, Electrical and Computer Engineering Department, Albuquerque, USA
} 


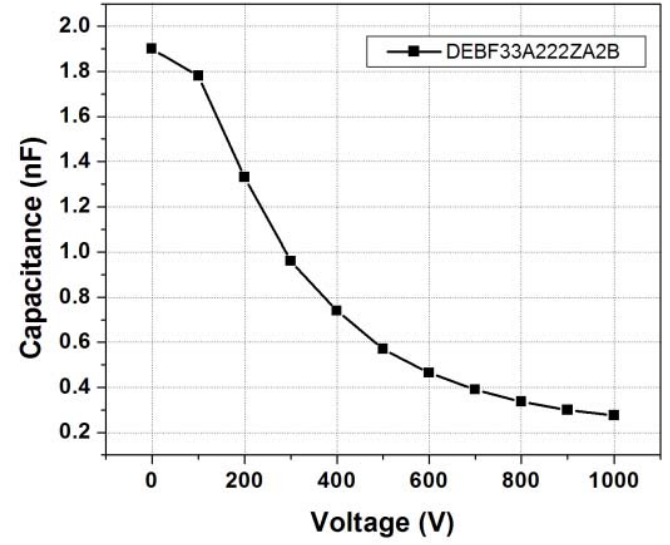

Figure 2. $\mathrm{C}-\mathrm{V}$ curve measured for the $2.2 \mathrm{nF}$ ceramic capacitor.

A commercial-off-the-shelf ferrite bead with nominal inductance of $10 \mu \mathrm{H}$ and part number 7427501 from RoHS maker, with wires wound on the ferrite core, was selected as a nonlinear inductor to build the hybrid line. To measure the inductance as a function of the applied current the circuit presented in Fig. 3 was used.

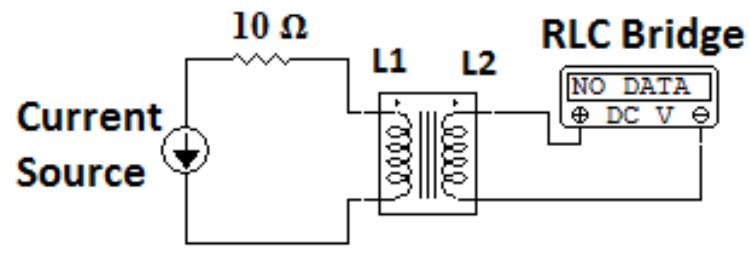

Figure 3. Circuit used for the characterization of the ferrite bead under DC condition.

As shown Fig. 4 the inductance exhibits a variation of the order of $71 \%$ on the L-I curve at $1 \mathrm{~A}$ current, whose result is very promising for use in hybrid lines for soliton RF generation.

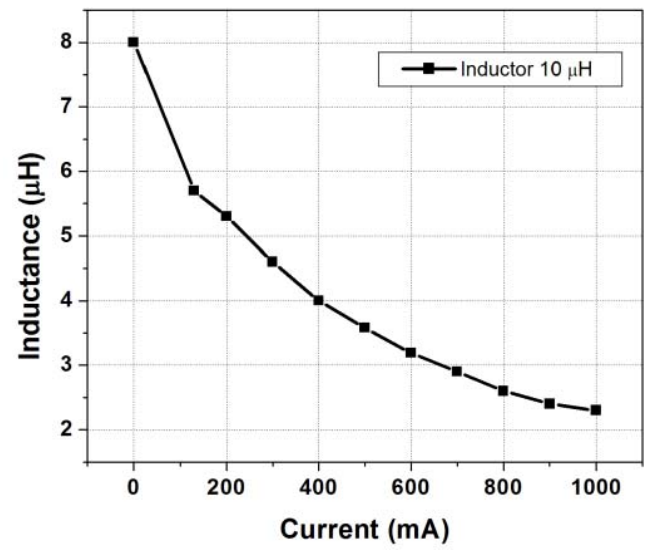

Figure 4. L-I curve measured for the $10 \mu \mathrm{H}$ ferrite bead.
The electric circuit used to produce a negative pump pulse at the input of line is shown in Fig. 5, where a pulse generator is used to trigger the driver and IGBT transistor to produce a discharge of capacitor bank (Cbank), resulting in a negative pump pulse at the input side of the line. The diodes D1 and D2 are used to protect the IGBT against reverse voltage and scape current respectively. The driver used to control the IGBT allows pump pulse with faster rise time.

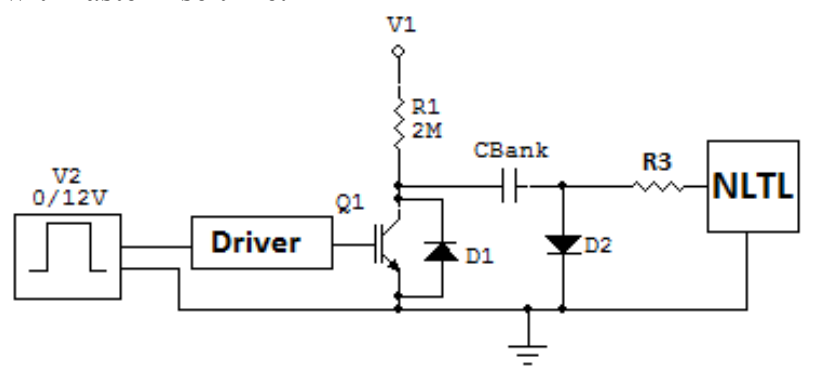

Figure 5. Electric circuit used to produce a negative pump input pulse into the line.

The 30-section hybrid line assembled on a printed circuit board (PCB) with $2.2 \mathrm{nF}$ ceramic capacitors and 10 $\mu \mathrm{H}$ ferrite beads is shown in Fig. 6, including a $75 \Omega$ load resistance put at the line output.

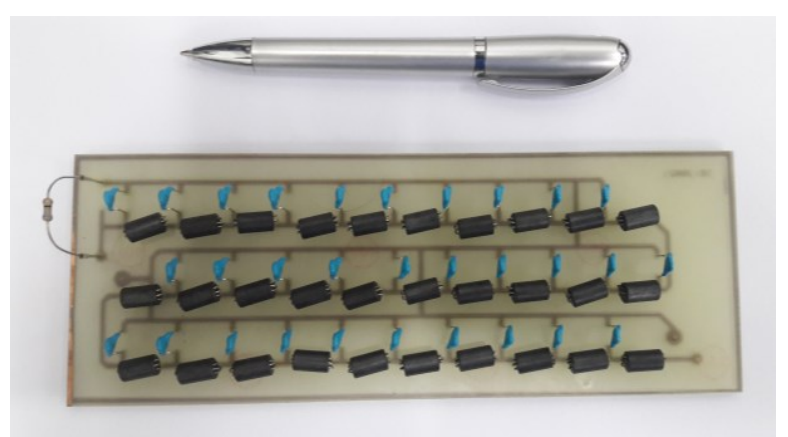

Figure 6. The HNLTL assembled on a PCB.

\section{III.RESULTS AND DISCUSSION}

Using the electric circuit of Fig. 5 and setting V1 to 300 $\mathrm{V}$, the soliton RF generated in the middle of line reached a frequency of order of $20 \mathrm{MHz}$ as shown in Fig. 7 and confirmed by the fast Fourier transform (FFT) exhibited in Fig. 8. The voltage modulation depth (VMD) obtained on the middle section was of about $290 \mathrm{~V}$ as shown in Fig. 7. For a $75 \Omega$ a load resistance and with $\mathrm{V} 1=300 \mathrm{~V}$, the current passing through the line is of the order of $4 \mathrm{~A}$. As the ferrite bead L-I characteristic was measured up to $1 \mathrm{~A}$ as seen in Fig. 4, then the inductance behavior was estimated through the oscillation frequency generated given by $f=1 / 2 \pi \sqrt{ }\left(\mathrm{LC}\left(\mathrm{V}_{\max }\right)\right)$. With $\mathrm{C} \approx 1.0 \mathrm{nF}$ at $\mathrm{V}_{\max }=$ $300 \mathrm{~V}$ (see Fig. 2) and $f=20 \mathrm{MHz}, \mathrm{L}$ calculated is of about $66 \mathrm{nH}$ giving an inductance decrease of approximately $99 \%$, thus leading to the saturation of the ferrite bead. 


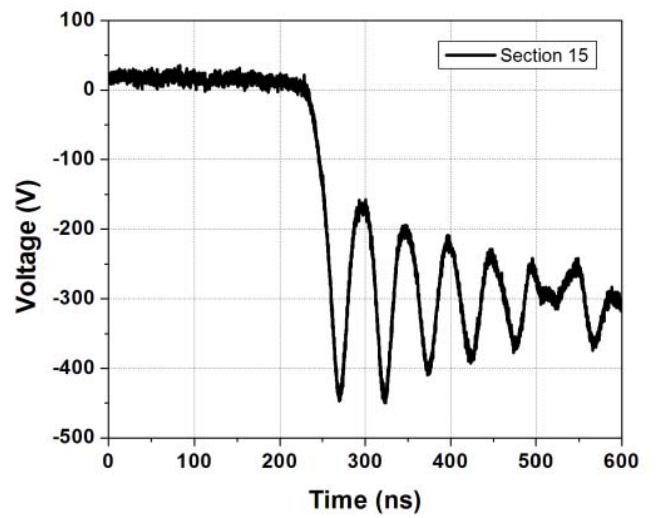

Figure 7. Soliton generated on the $15^{\text {th }}$ section of the HNLTL.

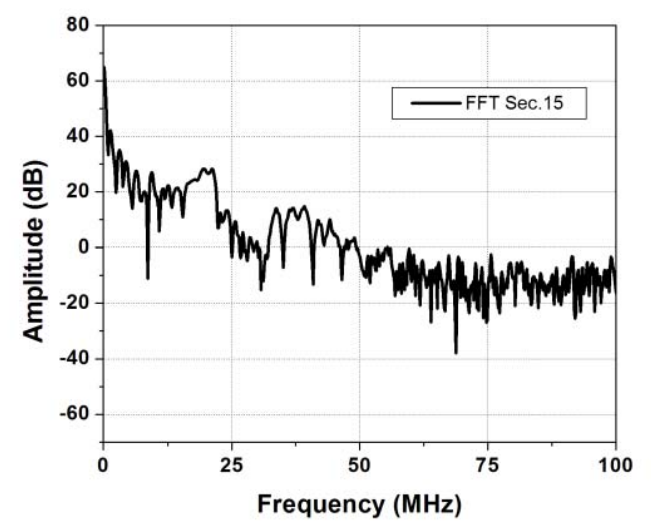

Figure 8. FFT obtained for $15^{\text {th }}$ section in the HNLTL.

On the other hand, the soliton RF waveform measured on the load resistance of $75 \Omega$ has the highest peak voltage of the order of $600 \mathrm{~V}$ as well as the lowest VMD of about $130 \mathrm{~V}$ as seen in Fig. 9. This is explained by the fact that the load resistance is never matched to the varying nonlinear characteristic impedance of the line. In this case, the low number cycles observed is also due to the to the load mismatch, which causes strong VMD attenuation.

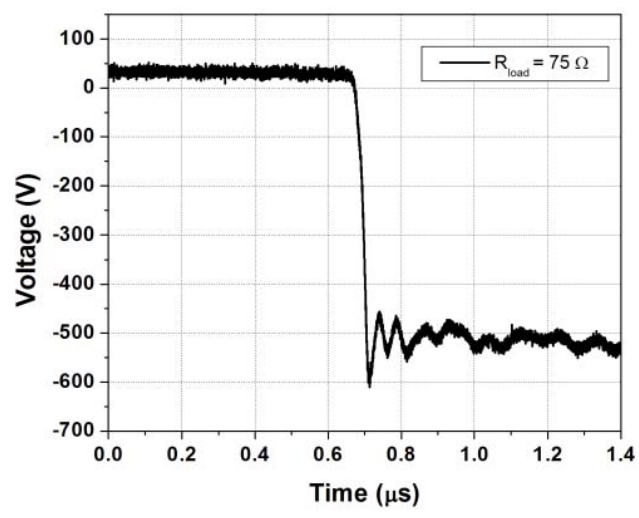

Figure 9. Soliton generated at the load resistance.
The FFT spectrum measured at the load confirms the soliton generated frequency at the order of $20 \mathrm{MHz}$ as shown in the Fig. 10.

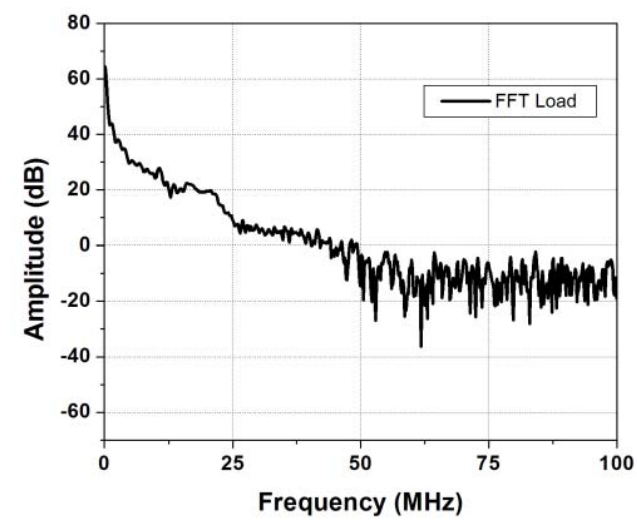

Figure 10. FFT obtained for load resistance.

\section{IV.CONCLUSIONS}

Commercial-off-the-shelf ceramic capacitors and ferrite beads with nonlinear behavior as a function of voltage and current applied, respectively, were used to build the experimental line for the second time up to knowledge of the authors so far. As found in the literature on HNLTLs, the first experimental result was obtained by Kuek et al., who [3] reported soliton RF of about $60 \mathrm{MHz}$, using a similar line with ferrite bead inductors and commercial ceramic capacitors. It was again demonstrated in our work RF soliton generation of about $20 \mathrm{MHz}$ using COTS ceramic capacitors with decrease in capacitance of the order of $85 \%$ near their nominal breakdown voltage, and ferrite beads with decay in inductance of the order of $99 \%$. In contrast to a positive polarity impulse described in the Kuek et al. experiment, we used a special system to produce a negative $\mathrm{HV}$ pump input pulse into the HNLTL. However, in our case we have obtained a soliton oscillation frequency lower than that generated by Kuek's line as ceramic capacitors were less stressed during pulse application due to the lower amplitude of the pump input pulse generated by our negative impulse system.

\section{V.REFERENCES}

[1] L. P. Silva Neto, J. O. Rossi, J. J. Barroso, E. Schamiloglu, "High- Power RF Generation from nonlinear transmission lines with barium titanate ceramic capacitors," IEEE Trans. Plasma Sci., vol. 44, no. 12, pp. 3424-3431, Dec. 2016.

[2] D. Yoshida, H. Ishizawa, T. Tanabe, K. Sugimoto, S. H. R. Hosseini, S. Katsuki and H. Akiyama, "Development of burst high frequency wave source for 
medical application," in Proc. of the IEEE International Pulsed Power Conference, 2013. p.1-4.

[3] N.S. Kuek, A.C. Liew, E. Schamiloglu, and J.O. Rossi, "RF pulse generator based on a nonlinear hybrid line," IEEE Trans. Plasma Sci., vol. 42, no. 10, pp. 32683273, Oct. 2014.

[4] L. P. Silva Neto, J. O. Rossi, J. J. Barroso, and A. R. Silva Junior, "Characterization of ceramic dielectrics for Sub-GHz applications in nonlinear transmission lines" IEEE Trans. Plasma Sci., vol. 42, no. 10, pp. 3274-3282, Oct. 2014. 